\section{Bortezomib in refractory ANCA-associated vasculitis: a new option?}

Bortezomib is a proteasome inhibitor approved for the treatment of multiple myeloma. Proteasome inhibition depletes short-lived and long-lived plasma cells and is a promising therapeutic approach to treat refractory antibody-mediated autoimmune diseases. In a small uncontrolled study from Charité (Berlin), recently published in the Annals of the Rheumatic Diseases, 12 patients with systemic lupus erythematosis (SLE) with persistent disease activity and autoantibody production despite immunosuppression were treated with bortezomib according to the approved protocol for multiple myeloma. ${ }^{1}$ Disease activity declined significantly, though adverse events occurred in 11 of 12 patients and resulted in discontinuation of bortezomib in seven of them.

Resistance to immunosuppressive treatment, including cyclophosphamide (CYC) and B-cells depletion, may be a challenge in SLE and in antineutrophil cytoplasmic autoantibodies (ANCA)-associated vasculitides. Rituximab is highly effective (82\%-100\%) for remission induction or maintenance treatment in patients with relapsing or refractory ANCA-associated vasculitis. ${ }^{2}$ Nevertheless, a few patients do not respond to this agent and therefore require additional options. Bontscho et $a l^{3}$ in an experimental study showed that bortezomib depleted myeloperoxidase (MPO)-specific plasma cells and prevented anti-MPO IgG-mediated necrotising crescentic glomerulonephritis in mice. A search in Pubmed revealed no case reports of bortezomib treatment in patients with ANCA-associated vasculitis.

We observed a 58-year-old woman with ANCA-associated vasculitis classified as microscopic polyangiitis who presented with purpura, increased serum creatinine (up to $268 \mu \mathrm{mol} / \mathrm{L}$ ), microhaematuria, mild proteinuria and high antiproteinase 3-ANCA titre (207 U/L). There were no signs of lung or upper respiratory tract disease. Kidney biopsy showed mesangioproliferative glomerulonephritis with fibrotic crescents. Induction treatment with CYC and high-dose glucocorticoids and later two rituximab infusions ( $1 \mathrm{~g}$ with 2 weeks' interval) was only partly effective, while one cycle of bortezomib (four subcutaneous injection of $1.3 \mathrm{mg} / \mathrm{m}^{2}$ with weekly intervals) without dexamethasone has induced prolonged remission of kidney disease and allowed to discontinue glucocorticoids. At 4 years of follow-up, kidney function was stable and there were no signs of activity of vasculitis without any maintenance treatment.

In the study by Alexander et al, patients with SLE received one to four (median 2) cycles of intravenous bortezomib \pm dexamethasone $20 \mathrm{mg}$ and restarted maintenance therapy after a median of 41 days (range 1-61 days) following the last bortezomib infusion. Our case shows that in autoimmune disease treatment with bortezomib may be probably less aggressive than in multiple myeloma. Intensity of plasma cells depletion is important as a possible benefit of bortezomib should be weighted against side effects (eg, infections and neuropathy) that occurred in $92 \%$ of patients with lupus and resulted in premature discontinuation in $58 \%$ of patients. In our patient, subcutaneous bortezomib injections were well tolerated and were not associated with any side effects.

ANCA-associated vasculitis is frequently a life-threatening or organ-threatening disease that may be refractory to conventional treatment. Introduction of rituximab was a major achievement, but we still need other agents for a relatively small proportion of patients who do not respond to or are intolerant of modern treatment. It seems that bortezomib constitutes a new putative therapeutic option for selected patients both with SLE and ANCA-associated vasculitis (and probably other serious antibody-mediated diseases). However, additional data are obviously needed.

\section{Pavel Novikov, Sergey Moiseev, Nikolay Bulanov, Elena Shchegoleva}

Clinic of Nephrology, Internal and Occupational Diseases, Sechenov First Moscow State Medical University, Moscow, Russia

Correspondence to Professor Sergey Moiseev, Clinic of Nephrology, Internal and Occupational Diseases, Sechenov First Moscow State Medical University, Rossolimo, 11/5, Moscow 119435, Russia; clinpharm@mtu-net.ru

Contributors All authors contributed to follow-up of the patient and review of the manuscript.

Competing interests None declared.

Ethics approval Retrospective data in patient treated in real-life practice.

Provenance and peer review Not commissioned; internally peer reviewed.

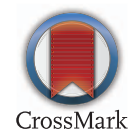

To cite Novikov P, Moiseev S, Bulanov N, et al. Ann Rheum Dis 2016;75:e9.

Received 19 May 2015

Accepted 21 May 2015

Published Online First 21 July 2015

Ann Rheum Dis 2016;75:e9. doi:10.1136/annrheumdis-2015-207947

\section{REFERENCES}

1 Alexander T, Sarfert R, Klotsche J, et al. The proteasome inhibitor bortezomib depletes plasma cells and ameliorates clinical manifestations of refractory systemic lupus erythematosus. Ann Rheum Dis 2015;74:1474-8.

2 Alberici F, Jayne DR. Impact of rituximab trials on the treatment of ANCA-associated vasculitis. Nephrol Dial Transplant 2014;29:1151-9.

3 Bontscho J, Schreiber A, Manz RA, et al. Myeloperoxidase-specific plasma cell depletion by bortezomib protects from anti-neutrophil cytoplasmic autoantibodies-induced glomerulonephritis. J Am Soc Nephrol 2011;22:336-48. 